\title{
Screening for Obstructive Sleep Apnea Syndrome in the Preoperative Patients
}

\author{
Frances Chung*
}

Department of Anesthesia, University of Toronto, Toronto Western Hospital, University Health Network, Toronto, Ontario, Canada

\begin{abstract}
Obstructive sleep apnea (OSA) is caused by repetitive partial or complete obstruction of the upper airway, characterized by episodes of breathing cessation during sleep, which last 10 or more seconds. The prevalence of OSA varies widely depending on the demographics of the population studied, the definition of the disorder and the methods of diagnosis. Patients with obstructive sleep apnea can be a potential management challenge for the anesthesiologists during the perioperative period. Therefore anesthesiologists need a thorough understanding of the diagnosis of OSA, prevalence of OSA and the available screening tools.
\end{abstract}

Keywords: Obstructive sleep apnea, screening tools, anesthesia, STOP-Bang questionaire.

\section{DIAGNOSIS OF OSAS}

The diagnosis of OSA is established by an overnight sleep study or polysomnography. The apnea hyponea index (AHI) is the number of abnormal respiratory events per hour of sleep. There are different published clinical diagnostic criteria for OSA. In 1999, the task force of the American Academy of Sleep Medicine established the minimal clinical diagnostic criteria for OSA as an AHI of 10 plus symptoms of excessive daytime sleepiness [1]. The Canadian Thoracic Society guidelines stipulate the diagnostic criteria for OSA as having daytime sleepiness not explained by other factors or at least 2 other symptoms of OSA, with an AHI of 5 or more on polysomnography [2].

AHI cutoffs have been used to describe the severity of OSA. Mild OSA is defined as AHI between 5 and 15, moderate OSA as AHI between 15 and 30 and severe OSA as AHI more than 30 [3].

\section{PREVALENCE OF OBSTRUCTIVE SLEEP APNEA SYNDROME}

In the general population, moderately severe OSA (AHI $>15$ ) is present in $11.4 \%$ of men and $4.7 \%$ of women respectively $[4,5]$. In 1993, an epidemiologic study showed that $24 \%$ of male and $9 \%$ of female has AHI $>5$ respectively [6]. The prevalence of obstructive sleep apnea in four epidemiologic studies of the general population is shown in Table $\mathbf{1}$ $[7,8]$.

No epidemiologic studies have been conducted to determine the prevalence of OSA in the general population. However, a few studies have used screening questionnaire or polysomnography to determine the frequency of OSA in the general surgical population. We have studied patients

*Address correspondence to this author at the Department of Anesthesia, University of Toronto, Toronto Western Hospital, University Health Network, Toronto, Ontario, Canada; E-mail: Frances.chung@uhn.on.ca undergoing elective surgery and $24 \%$ were identified preoperatively at high risk for OSA using the Berlin questionnaire [9]. We also screened 2467 patients using STOP questionnaire, $27.5 \%$ of them were classified as being at high risk of OSA [10]. Another study of patients identified 10 cases of undiagnosed OSA out of 254 patients undergoing joint arthroplasty by asking patients to complete a few screening questions [11].

In our recent study, we approached 2,467 surgical patients for preoperative polysomnography. Only two hundred and eleven patients underwent polysomnography and $69 \%$ were found to have AHI $>5$ [10]. Fidan et al., screened 433 surgical patients; only 18 of 41 invited patients agreed to undergo polysomnographic testing, and 14 patients $(3.2 \%$ of all screened patients) were diagnosed with OSA [12]. In a recent study by Finkel et al., [13], 661 of 2,778 patients undergoing elective surgery were identified high risk of OSA in preoperative clinics, where 534 of these were previously undiagnosed. Home sleep studies were performed and OSA was diagnosed in 207 patients using ARES Unicorder.

In studies of surgical population, the refusal rate for polysomnography was high. Self-selection from patients may exist because patients who had sleep symptoms might have selectively consented to the overnight polysomnography. The high refusal rate and dropout rate also indicate the difficulty that the study of prevalence in the general surgical population faced. This may be due to the anxiety about the upcoming surgery and the need to stay one night in the sleep laboratory or doing the sleep studies at home. The high prevalence of OSA in the group of surgical patients who underwent polysomnography may reflect this self-selection.

In selective surgical population, the prevalence of OSA is substantially higher than the incidence in the general population. In bariatric surgery, 7 out of every 10 patients were found to have OSA [14]. There is also a higher incidence of 
Table 1. Prevalence of Obstructive Sleep Apnea (OSA) in the General Population

\begin{tabular}{|c|c|c|c|c|c|c|}
\hline \multirow[b]{2}{*}{$\begin{array}{c}\text { Study } \\
\text { Location \& } \\
\text { Population }\end{array}$} & \multirow[b]{2}{*}{ Numbers (n) } & \multirow[b]{2}{*}{$\begin{array}{l}\text { Age Range } \\
\text { (Years) }\end{array}$} & \multicolumn{2}{|c|}{$\begin{array}{l}\text { Estimated Prevalence (\%) of Mild OSA } \\
(\mathrm{AHI} \geq 5)[95 \% \mathrm{CI}]\end{array}$} & \multicolumn{2}{|c|}{$\begin{array}{l}\text { Estimated Prevalence (\%) of Moderate OSA } \\
\qquad(\mathrm{AHI} \geq \mathbf{1 5})[95 \% \mathrm{CI}]\end{array}$} \\
\hline & & & Males & Females & Males & Females \\
\hline $\begin{array}{c}\text { Wisconsin [6] } \\
\text { (state employees) }\end{array}$ & $\begin{array}{c}602 \\
M=352 \\
F=250\end{array}$ & $30-60$ & $24(19-28)$ & $9(6-12)$ & $9(6-11)$ & $4(2-7)$ \\
\hline $\begin{array}{c}\text { Pennsylvania }[4,5] \\
\text { (household population) }\end{array}$ & $\begin{array}{c}1741 \\
M=741 \\
F=1000\end{array}$ & $20-100$ & $17(15-20)$ & - & $7(6-9)$ & $2(2-3)$ \\
\hline $\begin{array}{c}\text { Spain [8] } \\
\text { (general population) }\end{array}$ & $\begin{array}{c}2148 \\
M=1050 \\
F=1098\end{array}$ & $30-70$ & $26(20-32)$ & $28(20-35)$ & $14(10-18)$ & $7(3-11)$ \\
\hline
\end{tabular}

Legend: The above table is based on four studies with similar design and methodology that look at AHI among various adult populations. M=male, $\mathrm{F}=$ female. Adapted from SA Chung, Anesth Analg 2008; 107: 1543-63.

obstructive sleep apnea in medically refractory epilepsy patients requiring surgical intervention. The incidence is $33 \%$ [15]. In patients coming for intracranial tumour excision, the incidence of AHI $>5$ was $64 \%$ and the incidence of $\mathrm{AHI}>20$ was $55 \%[16]$.

\section{SCREENING TOOLS FOR OBSTRUCTIVE SLEEP APNEA IN THE SURGICAL SETTING}

The gold standard for the diagnosis of OSA is by overnight polysomnography. Polysomnography is expensive to perform, requiring highly trained personnel, sophisticated equipment and an entire night of recording. The long waiting lists for polysomnography in sleep centers have created interest for screening tools for obstructive sleep apnea. Therefore there is a need of a practical screening tool to identify patients more likely to have true OSA. In this way, polysomnography may be used in a cost-effective way for those patients who stand the most to gain from the investigations. There are 2 recent systematic reviews of screening instruments for obstructive sleep apnea by Abrishami et al., and Ramachandran et al., $[17,18]$. Both recommended STOP or STOP-Bang questionnaire as the optimal screening tool based on ease of use, false negative rate, and test accuracy. STOP-Bang is a user-friendly and excellent method to predict severe OSA (AHI $>30)$ with a diagnostic odds ratio of 142 [18].

\section{THE STOP OR STOP-BANG QUESTIONNAIRE}

The STOP questionnaire was developed and validated in surgical patients to facilitate the widespread usage of an OSA screening tool. The STOP questionnaire contains four questions and has an easy acronym: S-"Do you snore loudly, loud enough to be heard through closed door?"; T-"Do you feel tired or fatigued during the daytime almost every day?"; O-"Has anyone observed that you stop breathing during sleep?"; and P-"Do you have a history of high blood pressure with or without treatment?" (Table 2) $[10,19]$. In order to make it simple, the questions were designed in a yes/no format. A positive score of two "Yes" out of the four questions will indicate that the patient may be at high risk of OSA. The sensitivity of the STOP questionnaire at an AHI $>5,>15$ and $>30$ was $65.6,74.3$ and $79.3 \%$ respectively. The specificity at an AHI $>5,>15$ and $>30$ was $60.0,53.3$ and $48.6 \%$ respectively (Table 3) [10].

The STOP-Bang questionnaire is a scoring model consisting of 8 easily administered questions starting with the acronym STOP-BANG (Table 4) and is scored based on Yes/No. Thus, this can range from a value of 1-8, depending on how many questions have a 'yes' as answer. A patient is considered to be at high risk of OSA if $\geq 3$ items are scored positive on the STOP-Bang questionnaire [10]. The diagnostic ability of the STOP-Bang questionnaire was found to have a sensitivity of $93 \%$ and $100 \%$ at Apnea Hypopnea Index

Table 2. STOP Questionnaire

\begin{tabular}{|c|c|c|c|}
\hline & Questions & \multicolumn{2}{|c|}{ Answer } \\
\hline $\mathrm{T}$ & Tired: Do you often feel tired, fatigued, or sleepy during daytime? & Yes, & No \\
\hline $\mathrm{O}$ & Observed: Has anyone observed you stop breathing during your sleep? & Yes, & No \\
\hline $\mathrm{P}$ & Blood pressure: Do you have or are you being treated for high blood pressure? & Yes & No \\
\hline
\end{tabular}

High risk of OSA, answering yes to two or more questions. Low risk of OSA, answering yes to less than two questions. Adapted from Chung et al., Anesthesiology 2008; 108: 812-821. 
Table 3. Predictive Parameters of STOP, STOP-BANG, Berlin and ASA Questionnaires

\begin{tabular}{|c|c|c|c|c|}
\hline & STOP & STOP-BANG & Berlin & ASA \\
\hline \multicolumn{5}{|l|}{$\mathrm{AHI}>5$} \\
\hline Sensitivity\% & 65.6 & 83.6 & 68.9 & 72.1 \\
\hline Specificity $\%$ & 60.0 & 56.4 & 56.4 & 38.2 \\
\hline PPV\% & 78.4 & 81.0 & 77.9 & 72.1 \\
\hline NPV\% & 44.0 & 60.8 & 44.9 & 38.2 \\
\hline Odds ratio & 2.857 & 6.587 & 2.855 & 1.559 \\
\hline \multicolumn{5}{|l|}{$\mathrm{AHI}>15$} \\
\hline Sensitivity\% & 74.3 & 92.9 & 78.6 & 78.6 \\
\hline Specificity\% & 53.3 & 43.0 & 50.5 & 37.4 \\
\hline PPV\% & 51.0 & 51.6 & 50.9 & 45.1 \\
\hline NPV\% & 76.0 & 90.2 & 78.3 & 72.7 \\
\hline Odds ratio & 3.293 & 9.803 & 3.736 & 2.189 \\
\hline \multicolumn{5}{|l|}{$\mathrm{AHI}>30$} \\
\hline Sensitivity $\%$ & 79.5 & 100 & 87.2 & 87.2 \\
\hline Specificity\% & 48.6 & 37.0 & 46.4 & 36.2 \\
\hline PPV\% & 30.4 & 31.0 & 31.5 & 27.9 \\
\hline NPV\% & 89.3 & 100 & 92.8 & 90.9 \\
\hline Odds ratio & 3.656 & $>999.999$ & 5.881 & 3.862 \\
\hline
\end{tabular}

Data are presented as mean. AHI = apnea-hypopnea index; ASA=American Society of Anesthesiologists; NPV= negative predictive value: PPV= positive predictive value. Adapted from Chung et al., Curr Opin Anesthesiology 2009; 22:405-11.

Table 4. STOP-Bang Scoring Model

\begin{tabular}{|c|c|c|c|}
\hline & & \multicolumn{2}{|c|}{ Answer } \\
\hline S & Snoring: Do you snore loudly (louder than talking or loud enough to be heard through closed doors)? & Yes & No \\
\hline $\mathrm{T}$ & Tired: Do you often feel tired, fatigued, or sleepy during daytime? & Yes & No \\
\hline $\mathrm{O}$ & Observed: Has anyone observed you stop breathing during your sleep? & Yes & No \\
\hline $\mathrm{P}$ & Blood Pressure: Do you have or are you being treated for high blood pressure? & Yes & No \\
\hline B & BMI: $B$ MI more than $35 \mathrm{~kg} / \mathrm{m}^{2}$ & Yes & No \\
\hline A & Age: $A$ ge over 50 yr old & Yes & No \\
\hline $\mathrm{N}$ & Neck circumference: $N$ eck circumference greater than $40 \mathrm{~cm}$ & Yes & No \\
\hline G & Male & Yes & No \\
\hline
\end{tabular}

High risk of OSA, answering yes to three or more items. Low risk of OSA, answering yes to less than three items. Adapted from Chung, F et al. Anesthesiology 2008; 108: 812-821.

(AHI) cut-offs of $>15$ and $>30$, respectively, with a specificity of $43 \%$ and $37 \%$, respectively (Table 4) [10]. The STOPBang questionnaire has a very high level of sensitivity and negative predictive value, especially for patients with moderate and severe OSA. If the patient is ranked as a low risk of OSA by the STOP-Bang questionnaire, we could be highly confident about excluding that the patient would have moderate to severe OSA. For safety reasons, the screening tool should have a high degree of sensitivity at the expense of lower specificity. This means that STOP or STOP-Bang questionnaire may have false positive results. Patients that are identified with high risk of OSA on STOP-Bang questionnaire has been shown to be associated with the occurrence of higher postoperative complications [20].

There are a number of screening tools in the literature $[6$, 21-25] e.g. Wisconsin questionnaire, the Sleep Disorder Questionnaire (SDQ), Hawaii sleep questionnaire, Selfreport questionnaire by Haraldsson, Self-reported questionnaire by Pouliot et al., and modified Berlin questionnaire were all developed and tested in patients mainly from sleep centers. Flemons has developed a prediction questionnaire for obstructive sleep apnea. In this model, the clinical prob- 
ability that a sleep test (PSG or home monitoring) would be positive for OSA was based on the adjusted neck circumference [26]. Patients referred to sleep centers are suspected of having sleep related disorders especially OSA. They are preselected patients, therefore screening tools for OSA developed and validated in sleep centers cannot be applied to other patient populations without validation in the target patient population [10].

The Berlin questionnaire is one of the commonly known questionnaires for OSA and has been validated in the patients in primary care setting [27]. It has 11 questions organizing into three categories. The predictive performance of the Berlin questionnaire varies in different patient population. We have shown that in the surgical population the sensitivity and specificity is similar to the STOP questionnaire [20]. However, the number of questions and the complicated scoring system may be too cumbersome for anesthesiologists and their patients.

The American Society of Anesthesiologists' checklist is 14-item questions to assist anesthesiologists in identifying OSA. The checklist is composed of three categories of predisposing physical characteristics, symptoms and complaints attributable to OSA. The sensitivity and specificity of the ASA checklist is similar to the STOP questionnaire [20].

Recently, a P-SAP score composed of 9 items: male gender, history of snoring, thick neck, modified mallampati class 3 or 4 , TM distance $<6 \mathrm{~cm}$, hypertension, type 2 diabetes mellitus, BMI $>30 \mathrm{~kg} / \mathrm{m} 2$ and age $>43$ was developed. P$\mathrm{SAP}>$ or equal to 4 has a sensitivity of 0.67 and a specificity of 0.77 [28].

\section{EVALUATION OF PATIENTS WITH SUSPECTED OSA IN THE PREOPERATIVE CLINIC}

It is estimated that $82 \%$ of men and $92 \%$ of women with moderate-to severe OSA have not been diagnosed [29]. From the anesthesiologists' viewpoint, patients with OSA may present significant problems in the perioperative period, including difficult airways, sensitivity to anesthetic agents, and postoperative adverse events. OSA has been associated with an increase in postoperative complications $[30,31]$ and is a risk factor for increased morbidity and mortality [32, 33].

We did a matched cohort study in elective surgical patients with OSA and found that patients with OSA had an increased incidence of postoperative complications with a hazard ratio of 2 [31]. In addition, there is literature showing that patients with OSA undergoing upper airway surgery [34, 35], joint replacement surgery [36], and cardiac surgery [37] have an increased risk of postoperative complications.

We have shown that patients who are screened positive for STOP questions are more likely to have postoperative complications $[10,20]$. These results were confirmed in a recent study that patients who are screened positive for STOP- Bang questionnaire are more likely to have postoperative complications versus those who are screened negative (19.3\% vs. $1.3 \% \mathrm{p}<0.001)$ [38].

Screening questionnaire like STOP-Bang should be used in preoperative clinic to help identify patients at risk of OSA. A patient is at high risk of OSA if 2 items or more score positive on the STOP questionnaire or 3 items or more score positive on the STOP-Bang questionnaire. Urgent or emergency surgery should not be delayed for the detailed evaluation of suspected OSA. Perioperative precautions are important. Protocols have been developed by the American Society of Anesthesiologists [39]. We also published a suggested protocol in the Canadian Journal of Anesthesia which is less arduous and requires less monitoring than the ASA guideline [40].

\section{CONCLUSIONS}

There is a high prevalence of undiagnosed OSA in the surgical patients. Sleep apnea is also associated with other pre-existing medical diseases such as obesity, hypertension, coronary artery disease, diabetes mellitus that may negatively impact outcomes. The patients with OSA pose special challenges to the anesthesiologist in the perioperative period. Preoperative screening and evaluation may ameliorate the perioperative morbidity associated with patients with OSA.

\section{REFERENCES}

[1] The Report of an American Academy of Sleep Medicine Task Forces. Sleep-related breathing disorders in adults: recommendations for syndrome definition and measurement techniques in clinical research. Sleep 1999; 22: 667-89.

[2] Fleetham J, Ayas N, Bradley D, et al. Canadian Thoracic Society guidelines: diagnosis and treatment of sleep disordered breathing in adults. Can Respir J 2006; 13: 387-92.

[3] Iber C, Ancoli-Israel S, Cheeson A, et al. The AASM manual for the scoring of sleep and associated events, rules, terminology and technical specifications. Westcherster (IL): American Academy of Sleep Medicine 2007.

[4] Bixler EO, Vgontzas AN, Ten HT, et al. Effects of age on sleep apnea in men: 1. Prevalence and severity. Am J Respir Crit Care Med 1998; 157: 144-8.

[5] Bixler EO, Vgontzas AN, Lin HM, et al. Prevalence of sleepdisordered breathing in women: effect on gender. Am J Respir Crit Care Med 2001; 163: 608-13.

[6] Young T, Palta M, Dempsey J, et al. The occurrence of sleepdisordered breathing among middle-aged adults. N Engl J Med 1993; 328: 1230-5.

[7] Chung SA, Yuan H, Chung F. A systemic review of obstructive sleep apnea and its implications for anesthesiologists. Anesth Analg 2008; 107: 1543-63.

[8] Duran J, Esnaola S, Rubio R, Iztueta A. Obstructive sleep apneahypopnea and related clinical features in a population based sample of subjects aged 30 to $70 \mathrm{yr}$. Am J Respir Crit Care Med 2001; 163: 685-9.

[9] Chung F, Ward B, Ho J, Yuan H, Kayumov L, Shapiro C. Preoperative identification of sleep apnea risk in elective surgical patients using the Berlin questionnaire. J Clin Anesth 2007; 19: 130-4.

[10] Chung F, Yegneswaran B, Liao P, et al. STOP questionnaire: a tool to screen patients for obstructive sleep apnea. Anesthesiology 2008; 108: 812-21.

[11] Harrison MM, Childs A, Carson PE. Incidence of undiagnosed sleep apnea in patients scheduled for elective joint arthroplasty. J Arthrop 2003; 18: 1044-7.

[12] Fidan H, Fidan F, Unlu M, Ela Y, Ibis A, Tetik L. Prevalence of sleep apnoea in patients undergoing operation. Sleep Breath 2006; 10: 161-5.

[13] Finkel KJ, Searleman AC, Tymkew H, et al. Prevalence of undiagnosed obstructive sleep apnea among adult surgical patients in an academic medical center. Sleep Med 2009; 10: 753-8.

[14] Frey WC, Pilcher J. Obstructive sleep-related breathing disorders in patients evaluated for bariatric surgery. Obes Surg 2003; 13: 676-83.

[15] Malow BA, Levy K, Maturen K, Bowes R. Obstructive sleep apnea is common in medically refractory epilepsy patients. Neurology 2000; 55: 1002-7. 
[16] Pollak L, Shpirer I, Rabey JM, Klein C, Schiffer J. Polysomnography in patients with intracranial tumours before and after operation. Acta Neurol Scand 2004; 109: 56-60.

[17] Abrishami A, Khajehdehi A, Chung F. A systematic review of screening questionnaires for obstructive sleep apnea. Can J Anesth 2010; 57: 423-38.

[18] Ramachandran SK, Josephs LA. A Meta-analysis of Clinical Screening Tests for Obstructive Sleep Apnea. Anesthesiology 2009; 110: 928-39.

[19] Chung F, Elsaid H. Screening for obstructive sleep apnea before surgery: why is it important? Curr Opin Anesthesiol 2009; 22: 40511.

[20] Chung F, Yegneswaran B, Liao P, et al. Validation of the Berlin questionnaire and American society of anesthesiologists checklist as screening tools for obstructive sleep apnea in surgical patients. Anesthesiology 2008; 108: 822-30.

[21] Douglass AB, Bornstein R, Nino-Murcia G, et al. The sleep disorders questionnaire. I: creation and multivariate structure of SDQ. Sleep 1994; 17: 160-7.

[22] Kapuniai LE, Andrew DJ, Crowell DH, Pearce JW. Identifying sleep apnea from self-reports. Sleep 1988; 11: 430-6.

[23] Haraldsson PO, Carenfelt C, Knutsson E, Persson HE, Rinder J. Preliminary report: validity of symptom analysis and daytime polysomnography in diagnosis of sleep apnea. Sleep 1992; 15: 261-3.

[24] Pouliot Z, Peters M, Neufeld H, Kryger MH. Using self-reported questionnaire data to prioritize OSA patients for polysomnography. Sleep 1997; 20: 232-6.

[25] Sharma SK, Vasudev C, Sinha S, Banga A, Pandey RM, Handa KK. Validation of the modified Berlin questionnaire to identify patients at risk for the obstructive sleep apnoea syndrome. Indian J Med Res 2006; 124: 281-90.

[26] Flemons WW. Obstructive sleep apnea. N Engl J Med 2002; 347: 498-504.

[27] Netzer NC, Stoohs RA, Netzer CM, Clark K, Strohl KP. Using the Berlin Questionnaire to identify patients at risk for the sleep apnea syndrome. Ann Intern Med 1999; 131: 485-91.

[28] Ramachandran SK, Kheterpal S, Consens F, et al. Derivation and validation of a simple perioperative sleep apnea prediction score. Anesth Analg 2010; 110: 1007-15.
[29] Ancoli-Israel S, Kripke DF, Klaube MR, et al. Sleep-disordered breathing in community-dwelling elderly. Sleep 1991; 14: 486-95.

[30] Hwang D, Shakir N, Limann B, et al. Association of sleepdisordered breathing with postoperative complications. Chest 2008; 133: 1128-34

[31] Liao P, Yegneswaran B, Vairavanathan S, et al. Postoperative complications in patients with obstructive sleep apnoea: a retrospective matched cohort study. Can J Anesth 2009; 56: 819-28.

[32] Young T, Finn L, Peppard PE, et al. Sleep disordered breathing and mortality: eighteen-year follow-up of the Wisconsin sleep cohort. Sleep 2008; 31: 1071-8.

[33] Marshall NS, Wong KKH, Liu PY, et al. Sleep apnea as an independent risk factor for all-cause mortality: the Busselton Health Study. Sleep 2008; 31: 1079-85.

[34] Kim JA, Lee JJ, Jung HH. Predictive factors of immediate postoperative complications after uvulopalatopharyngoplasty. Laryngoscope 2005; 115: 1837-40.

[35] Pang KP. Identifying patients who need close monitoring during and after upper airway surgery for obstructive sleep apnea. J Laryngol Otol 2006; 120: 655-60.

[36] Gupta RM, Parvizi J, Hanssen AD, et al. Postoperative complications in patients with obstructive sleep apnea syndrome undergoing hip or knee replacement: a case-control study. Mayo Clin Proc 2001; 76: 897-905.

[37] Kaw R, Golish J, Ghamande S, et al. Incremental risk of obstructive sleep apnea on cardiac surgical outcomes. J Cardiovasc Surg (Torino) 2006; 47: 683-9.

[38] Vasu TS, Doghramji K, Cavallazzi R, et al. Obstructive sleep apnea syndrome and postoperative complications: clinical use of the STOP-BANG questionnaire. Arch Otolaryngol Head Neck Surg 2010; 136: 1020-4.

[39] Gross JB, Bachenberg KL, Benumof JL, et al. Practice guidelines for the perioperative management of patients with obstructive sleep apnea: a report by the American Society of Anesthesiologists' Task Force on perioperative management of patients with obstructive sleep apnea. Anesthesiology 2006; 104: 1081-93.

[40] Seet E, Chung F. Management of sleep apnea in adults - functional algorithms for the perioperative period. Can J Anesth 2010; 57: 849-64. 